\title{
Frame dependence of ${ }^{3} \mathrm{He}$ transverse $\left(e, e^{\prime}\right)$ response functions at intermediate momentum transfers
}

\author{
Victor D. Efros, ${ }^{1}$ Winfried Leidemann, ${ }^{2,3}$ Giuseppina Orlandini, ${ }^{2,3}$ and Edward L. Tomusiak ${ }^{4}$ \\ ${ }^{1}$ Russian Research Centre "Kurchatov Institute”, 123182 Moscow, Russia \\ ${ }^{2}$ Dipartimento di Fisica, Università di Trento, I-38123 Trento, Italy \\ ${ }^{3}$ Istituto Nazionale di Fisica Nucleare, Gruppo Collegato di Trento, I-38123 Trento, Italy \\ ${ }^{4}$ Department of Physics and Astronomy, University of Victoria, Victoria, British Columbia, V8P 1A1 Canada
}

(Received 24 November 2010; published 13 May 2011)

\begin{abstract}
The transverse electron scattering response function of ${ }^{3} \mathrm{He}$ was recently studied by us in the quasielastic peak region for momentum transfers $q$ between 500 and $700 \mathrm{MeV} / c$. Those results, obtained using the active nucleon Breit (ANB) frame, are here supplemented by calculations in the laboratory, Breit, and ANB frames using the two-fragment model discussed in our earlier work on the frame dependence of the longitudinal response function $R_{L}(q, \omega)$. We find relatively frame-independent results and good agreement with experiment especially for the lower momentum transfers. This agreement occurs when we neglect an $\omega$-dependent piece of the one-body current relativistic correction. Inclusion of this term leads, however, to a rather pronounced frame dependence at $q=700 \mathrm{MeV} / c$. A discussion of this term is given here. This report also includes a correction to our previous ANB results for $R_{T}(q, \omega)$.
\end{abstract}

DOI: 10.1103/PhysRevC.83.057001

PACS number(s): 25.30.Fj, 21.45.-v, 21.30.-x

At momentum transfers reaching about $0.5 \mathrm{GeV} / c$ relativistic nuclear dynamics effects become pronounced in the electron scattering response functions. A relativistically covariant dynamics framework that might cope with those effects is not well defined at present, but work is in progress (see, e.g., [1]). In [2] we tried to incorporate the most significant relativistic features in a nonrelativistic calculation of the longitudinal response function. This was done by introducing the active nucleon Breit (ANB) reference frame. The laboratory frame response function was calculated by first computing that observable in the ANB frame and then transforming to the laboratory frame. The use of the ANB frame appeared to diminish the effects of using nonrelativistic nuclear dynamics. In addition, a two-fragment kinematical model was developed. In that model, one assumes a quasielastic knockout of a nucleon such that the residual nucleus remains in its lowest-energy state. Thus the energy transfer to the nucleus consists in the well-defined separation energy of the knocked-out nucleon and the kinetic energies of the fragments. Treating the kinematics of the fragments fully relativistically, one obtains the relativistic two-fragment relative momentum $p_{R}$ and kinetic energy $T_{R}$. In our model it is the momentum $p_{R}$ that is used to determine the corresponding nonrelativistic energy $T_{N R}$ via the nonrelativistic energy-momentum relation. We note that $T_{N R}>T_{R}$. This reflects the simple fact that in a nonrelativistic treatment one cannot have both the energy and momentum correct, i.e., the correct relativistic momentum $p_{R}$ used to determine the nonrelativistic energy employed in the calculation does not give the correct relativistic energy.

In general, as pointed out in [2], one prefers to ensure the correct relativistic momenta of outgoing particles rather than the correct total energy. Considering for example the laboratory frame, in the peak the quasielastically knocked-out nucleon has momentum $q$; thus one has a difference between nonrelativistic and relativistic kinetic energies of $\Delta T=$ $q^{2} / 2 M-\left(M^{2}+q^{2}\right)^{1 / 2}+M(M$ is the nucleon mass). Taking for example $q=700 \mathrm{MeV} / c$ one has $\Delta T=29 \mathrm{MeV}$. In the two-fragment model this relativistic effect is automatically taken into account. In fact one uses the correct $p_{R}$, and thus implicitly the correct $T_{R}$, while for the nonrelativistic calculation $T_{N R}$ is used. Since $T_{N R}=T_{R}+\Delta T$ one finds the correct quasielastic peak position.

It is important to state that this model only fixes a kinematical input while the full three-nucleon dynamics is still treated completely. In this respect, treatment of nucleon momenta in the two-fragment model resembles their treatment when fitting $N N$ data at an energy $T_{\text {lab }}$ for construction of an $N N$ interaction. In fact one has $\left(p_{R}^{\text {c.m. }}\right)^{2}=M T_{\text {lab }} / 2$ with $T_{N R}^{\text {c.m. }}=\left(p_{R}^{\text {c.m. }}\right)^{2} / M$ to be used in the Schrödinger equation. One could try to take into account that the interaction is defined in the $N N$ c.m. system and approximate this case as much as possible in a many-body calculation by making appropriate boosts. However, this is not considered in the present work.

Of interest is the point about the frame dependence of the response function calculation. The two-fragment model may be applied in various reference frames, and the laboratory frame results sought for may be obtained from results in other reference frames via a simple kinematics transformation [see Eq. (5) in [3]]. Use of results referring to various reference frames would lead, of course, to the same laboratory frame result if a relativistically covariant theory is employed. But in the framework of the "quasirelativistic" two-fragment model approach (relativistic momentum $p_{R}+$ nonrelativistic dynamics), this is possible only if this approach accounts properly for all essential relativistic features. Thus a comparison of calculations done in the ANB frame with those done in any other frame (including the ANB frame) but with the two-fragment kinematical model provides some indication of uncertainties due to noncovariance. This comparison in the longitudinal case [2] has demonstrated nearly a frame independence.

In a recent publication Ref. [3] we presented calculations of $R_{T}(q, \omega)$, the transverse electron scattering response function of ${ }^{3} \mathrm{He}$. That calculation was based on the Lorentz integral 
transform (LIT) technique [4-6] and included the AV18 [7] and $N N+$ UrbanaIX [8] $N N N$ potentials, the lowest-order relativistic corrections to the one-body and meson exchange currents (MECs) that are consistent with the AV18 interaction. (The two-body currents are of minor importance in our kinematics and they did not include relativistic corrections.) Detailed references to these aspects can be found in [3]. The laboratory frame response function was calculated with use of the ANB frame. In the present Brief Report we first correct those results and improve them due to use of the relativistic two-fragment model. Second, we study the frame dependence of the results for $R_{T}(q, \omega)$ when the two-fragment model is applied in various reference frames.

In dealing with the transverse response function of ${ }^{3} \mathrm{He}$ near the quasielastic peak where the energy-momentum of the virtual photon is absorbed predominantly by the single ejected nucleon, one can again adopt the two-fragment model. Compared to the longitudinal response function there is, however, a slight difference in applying the two-fragment model to the transverse response. For the former we did not include a two-body charge operator, while for the latter we do consider, as mentioned above, an MEC. It is not evident that such a two-body current should be taken into account in the two-fragment model, since it violates the assumption of a quasielastic knockout. Instead one may assume the following scenario (laboratory system): Initially two nucleons have opposite and equal momenta $\mathbf{p}$ and $-\mathbf{p}$ while the third nucleon is at rest. If the photon momentum is transferred to the 1-2 pair then their momenta in the final state become $\mathbf{p}+\mathbf{q} / 2$ and $-\mathbf{p}+\mathbf{q} / 2$. Setting the total kinetic energy energy of the 1-2 pair equal to $q^{2} / 2 M$, i.e., the excitation energy of the quasielastic peak, one can solve for $\mathbf{p}$ and hence the kinetic energy of the 1-2 pair using either relativistic or nonrelativistic kinematics. If one takes $q=700 \mathrm{MeV} / c$ then one can show that this difference varies between $16 \mathrm{MeV}$ (p and $\mathbf{q}$ orthogonal) and $29 \mathrm{MeV}$ (p and $\mathbf{q}$ parallel). As long as MEC matrix elements are small their main contribution arises from interference with the one-body current operator. Thus most probably their most significant effect is in the forward direction ( $\mathbf{p}$ and $\mathbf{q}$ parallel), which is the assumption of the two-fragment model. If, however, MEC and one-body current contributions are similar in size, a part of the MEC contribution is not taken into account with the proper final-state energy in the two-fragment model. Even then one may assume in most cases that the MEC changes only mildly with energy and thus errors remain small. Moreover, as already pointed out in [3] MECs have only a minor effect in the peak region.

In principle one should also consider implicit MEC contributions, which arise for example by application of the Siegert theorem. Although here we do not make use of the classical Siegert operators we need to better investigate the actual form of our one-body current. It is described in Eqs. (11) to (16) of Ref. [3]. One of the pieces of this current, referred to here as the $\omega$-dependent piece, appears as $(\omega / M) \mathbf{j}_{\omega}$ where $\omega$ is the energy transferred to the nuclear system by the virtual photon and where

$$
\mathbf{j}_{\omega}=e^{i \mathbf{q} \mathbf{r}^{\prime}} \frac{G_{E}-2 G_{M}}{8 M}\left(\mathbf{q}+i \kappa[\sigma \times \mathbf{q}]+2 i\left[\sigma \times \mathbf{p}^{\prime}\right]\right) .
$$

Here $\kappa$ is given by

$$
\kappa=1+2 P_{i} / A q,
$$

$\mathbf{r}^{\prime}$ and $\mathbf{p}^{\prime}$ are the relative coordinates and momenta of a single particle, $P_{i}$ is the magnitude of the target momentum, and $A$ is the nucleon number of the target. This current contains an implicit MEC contribution. For example, for deuteron photodisintegration in [9] it was shown that the contribution due to the spin-orbit term is almost exclusively an MEC contribution. For quasielastic kinematics, however, one has a completely different situation. In this respect it is helpful to realize that the operator form of the spin-orbit current, i.e., $\boldsymbol{\sigma} \times \mathbf{q}$, is identical to that of the nonrelativistic spin current and thus, in the quasielastic region, should lead like the latter mainly to one-body knockout in the forward direction.

For the explicit MEC we have studied the interference with the one-body current. As is to be expected, we find a rather weak interference effect only. Nonetheless it is the interference, not the MEC by itself, which leads to the bulk of the MEC contribution. Thus we may conclude that for quasielastic kinematics MEC contributions are quite small and due to interference with the one-body current and hence can be safely included in the two-fragment model.

In Fig. 1 we show various results for $R_{T}$ in the ANB frame. These results are obtained by calculating in the ANB frame and then transforming to the laboratory frame. The effect of $\mathbf{j}_{\omega}$ is negligibly small in the ANB frame since $\kappa=0$ in this case and $\omega=0$ at the quasielastic peak; thus its contribution is

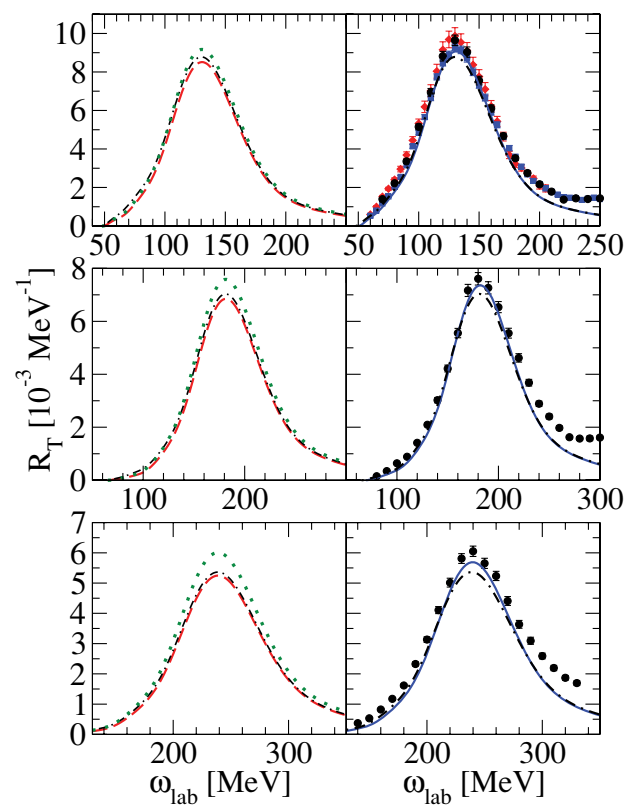

FIG. 1. (Color online) $R_{T}\left(q_{\mathrm{lab}}, \omega_{\mathrm{lab}}\right)$ at $q_{\mathrm{lab}}=500$ (top panels), 600 (middle panels), and $700 \mathrm{MeV}$ (bottom panels) $/ c$ from ANB frame calculation. In left-hand panels results without use of twofragment model with nonrelativistic one-body current (dotted), relativistic one-body current (dashed), and relativistic one-body current + MEC (dash-dotted). In right-hand panels results with relativistic one-body current + MEC without (dash-dotted) and with (solid) use of two-fragment model. Experimental data from [10] (squares), [11] (diamonds), and [12] (circles). 


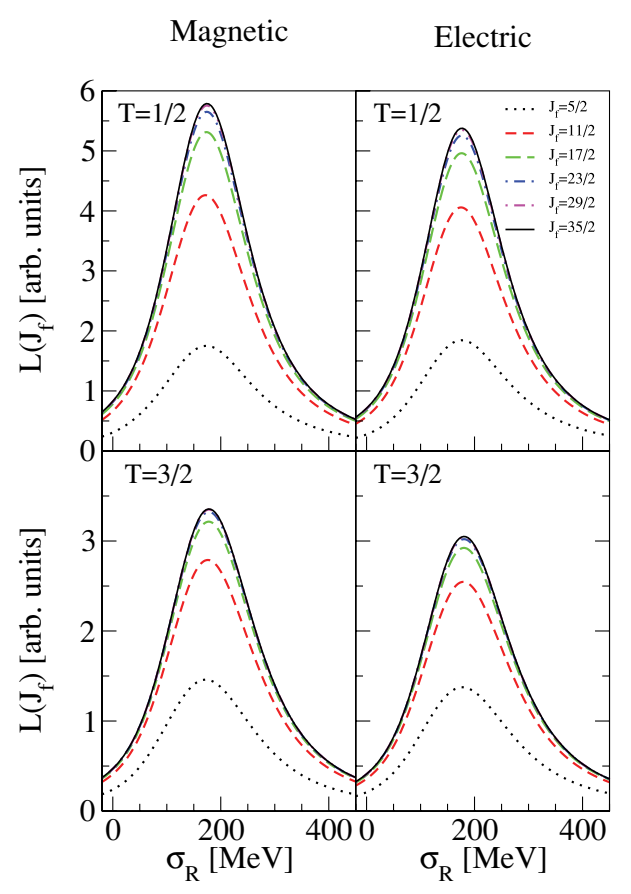

FIG. 2. (Color online) Convergence of Lorentz integral transforms with maximal final-state angular quantum number $J_{f}\left[L\left(J_{f}\right)\right]$ for magnetic (left panels) and electric (right panels) transition strength with final-state isospin quantum number $T_{f}=1 / 2$ (upper panels) and $3 / 2$ (lower panels); the LIT resolution parameter $\sigma_{I}$ [6] is equal to $50 \mathrm{MeV}$.

not shown separately. The ANB frame results in the left-hand panels of Fig. 1 replace those of [3], where an error resulting from an incorrect form factor argument gave results higher than experiment. In the present corrected version the ANB results are now lower than experiment. In order to show that the difference does not result from an insufficient consideration of higher-multipole contributions we show in Fig. 2 convergence tests for electric and magnetic LIT strength for both isospin channels as a function of final-state multipolarity at $q=$ $700 \mathrm{MeV} / c$. It is clear that the results are essentially fully converged by $J_{f}=35 / 2$. As an additional accuracy test we calculated the non-energy-weighted sum rules for $R_{T}(q, \omega)$ with the nonrelativistic one-body current operator. Comparing with the well-known non-energy-weighted spin sum rule [13], we obtain $98.4 \%, 98.5 \%$, and $98.0 \%$ at $q=500,600$, and $700 \mathrm{MeV} / c$, respectively. It shows that the sum rule is quite well satisfied.

It is of interest to compare this ANB calculation with one where the ANB calculation uses the two-fragment approximation for setting the kinematics. The results of this are depicted in the right-hand panels of Fig. 1 where one notes some difference between the two concerning the peak height only. Indeed, whereas in other frames the two-fragment model is effective in obtaining the quasielastic peak in the correct position this occurs automatically in the ANB frame without the need for the two-fragment model. On the other hand, the proper treatment of relativistic kinematics leads to a slight increase of the peak heights, namely, by $3.3 \%, 4.6 \%$, and $6.1 \%$ at $q=500,600$, and $700 \mathrm{MeV} / c$, respectively. Such

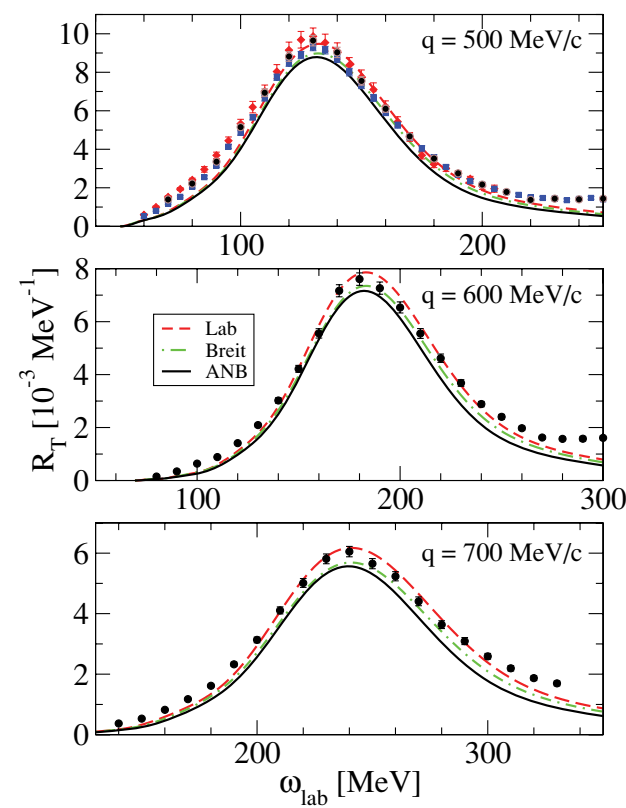

FIG. 3. (Color online) $R_{T}\left(q_{\text {lab }}, \omega_{\text {lab }}\right)$ at $q_{\text {lab }}=500,600$, and $700 \mathrm{MeV} / c$ with relativistic one-body current less $j_{\omega}$ with use of two-fragment model from calculations in various frames, namely, ANB (solid), laboratory (dashed), and Breit (dot-dashed) frames. Data as in Fig. 1.

an increase improves the agreement with experimental data; however, the theoretical peak heights are still somewhat too low, but one should also take into account that some additional strength could come from currents involving the $\Delta$ resonance.

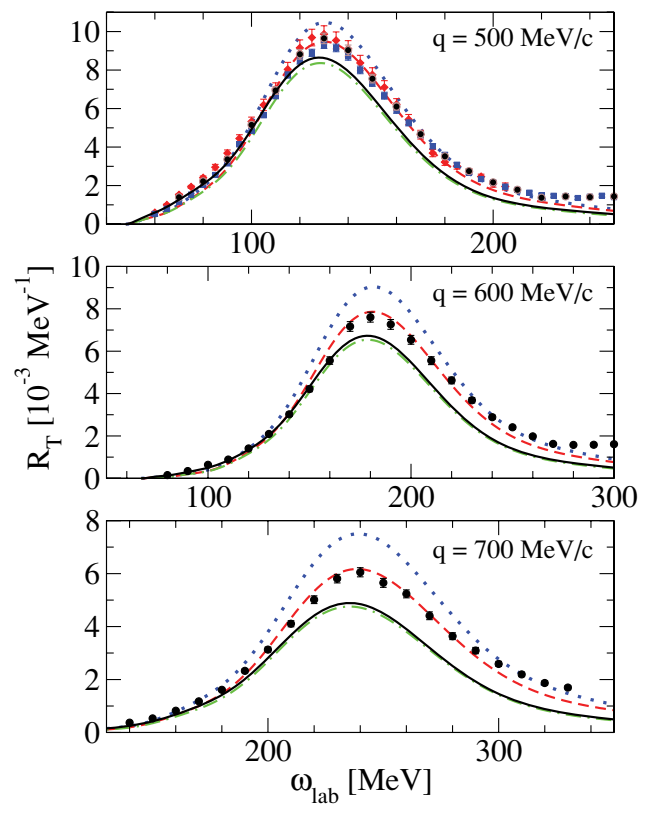

FIG. 4. (Color online) $R_{T}\left(q_{\text {lab }}, \omega_{\text {lab }}\right)$ at $q_{\text {lab }}=500,600$, and $700 \mathrm{MeV} / c$ from laboratory frame calculation with use of twofragment model: nonrelativistic one-body current (dotted), relativistic one-body current less $j_{\omega}$ (dashed), relativistic one-body current (dash-dotted), and relativistic one-body current + MEC (solid). Data as in Fig. 1. 
Our previous work [2] on frame dependence in $R_{L}(q, \omega)$ compared ANB frame results with those obtained in the laboratory and Breit frames all calculated using the two-fragment model. In Fig. 3 we show the corresponding results for $R_{T}(q, \omega)$ (MEC and $\mathbf{j}_{\omega}$ left out). As expected the experimental quasielastic peak position is reproduced in all three cases. For the quasielastic peak height one finds differences between $7 \%(q=500 \mathrm{MeV} / c)$ and $10 \%$ $(q=700 \mathrm{MeV} / c)$, showing an existing but not excessive frame dependence, which is about twice as large as the experimental error. The range of theoretical results covers the experimental data in the peak region.

Our conclusion concerning the relatively mild frame dependence relies on the assumption that contributions due to MEC and $\mathbf{j}_{\omega}$ remain small in other frames. While this assumption seems to be rather safe for the MEC it could be incorrect for $\mathbf{j}_{\omega}$, since it depends on the frame-dependent variables $\kappa$ and total energy transfer $\omega$. To better investigate this question we show in Fig. 4 the contributions of the various currents for the laboratory frame calculation using the two-fragment model. One can see that $\mathbf{j}_{\omega}$ has a significant effect on the laboratory system calculations and that the total reduction due to relativistic effects becomes very large (37\% at $q=$ $700 \mathrm{MeV} / c$ ). Comparing the result with the $\mathbf{j}_{\omega}$ contribution to the corresponding ANB frame results in the right-hand panels of Fig. 1, one finds reductions of the peak heights of about $5 \%(q=500 \mathrm{MeV} / c), 9 \%(q=600 \mathrm{MeV} / c)$, and $16 \%$ $(q=700 \mathrm{MeV} / c)$. Thus one has a rather pronounced frame dependence at $q=700 \mathrm{MeV} / c$. At this point we should remind the reader that the two-fragment model takes into account relativistic effects for the kinematics only, and that dynamical relativistic effects are not considered at all.

In such a situation with rather frame-dependent results one should choose the frame that leads to the smallest relativistic corrections. In [2] we already pointed that the ANB frame is preferable in the case of quasielastic kinematics, since in this frame the nucleon momenta in initial and final states are only of the order of $q / 2$, while in other frames one finds larger momenta, e.g., in the laboratory frame the knocked-out nucleon has momentum $q$. In fact the reduction of $R_{T}$ due to relativistic effects is relatively small in the ANB frame (13\% instead of the $37 \%$ in the laboratory frame at $q=700 \mathrm{MeV} / c$ ). Therefore we consider the ANB results to be more realistic than, e.g., the laboratory results. On the other hand it would be desirable to further reduce the frame dependence. This is possibly achieved by taking into account boost corrections. This could be done in a way similar to that used in deuteron electrodisintegration $[14,15]$.

Acknowledgments of financial support are given to the RFBR, Grant No. 10-02-00718 and RMES, Grant No. NS7235.2010.2 (V.D.E.), and to the National Science and Engineering Research Council of Canada (E.L.T.).
[1] S. A. Pinto, A. Stadler, and F. Gross, Phys. Rev. C 81, 014007 (2010).

[2] V. D. Efros, W. Leidemann, G. Orlandini, and E. L. Tomusiak, Phys. Rev. C 72, 011002(R) (2005).

[3] V. D. Efros, W. Leidemann, G. Orlandini, and E. L. Tomusiak, Phys. Rev. C 81, 034001 (2010).

[4] V. D. Efros, W. Leidemann, and G. Orlandini, Phys. Lett. B 338, 130 (1994).

[5] V. D. Efros, W. Leidemann, G. Orlandini, and E. L. Tomusiak, Phys. Rev. C 69, 044001 (2004).

[6] V. D. Efros, W. Leidemann, G. Orlandini, and N. Barnea, J. Phys. G 34, R459 (2007).

[7] R. B. Wiringa, V. G. J. Stoks, and R. Schiavilla, Phys. Rev. C 51, 38 (1995).
[8] B. S. Pudliner, V. R. Pandharipande, J. Carlson, S. C. Pieper, and R. B. Wiringa, Phys. Rev. C 56, 1720 (1997).

[9] P. Wilhelm, W. Leidemann, and H. Arenhövel, Few-Body Syst. 3, 111 (1988).

[10] C. Marchand et al., Phys. Lett. B 153, 29 (1985).

[11] K. Dow et al., Phys. Rev. Lett. 61, 1706 (1988).

[12] J. Carlson, J. Jourdan, R. Schiavilla, and I. Sick, Phys. Rev. C 65, 024002 (2002).

[13] G. Orlandini and M. Traini, Rep. Prog. Phys. 54, 257 (1991).

[14] G. Beck and H. Arenhövel, Few-Body Syst. 13, 165 (1992).

[15] F. Ritz, H. Göller, T. Wilbois, and H. Arenhövel, Phys. Rev. C 55, 2214 (1997). 